\title{
A moving crack in a rectangular magnetoelectroelastic body
}

\author{
Ke-Qiang $\mathrm{Hu}^{\text {a,b,* }}$, Yi-Lan Kang ${ }^{\text {a }}$, Qing-Hua Qin ${ }^{\mathrm{c}}$ \\ a Department of Mechanics, School of Mechanical Engineering, Tianjin University, Tianjin 300072, PR China \\ ${ }^{\mathrm{b}}$ Department of Civil, Environmental \& Ocean Engineering, Stevens Institute of Technology, Hoboken, NJ 07030, USA \\ ${ }^{c}$ Department of Engineering, Australian National University, Canberra, ACT 0200, Australia
}

Received 5 December 2005; received in revised form 20 April 2006; accepted 16 June 2006

Available online 30 August 2006

\begin{abstract}
The singular stress, electric fields and magnetic fields in a rectangular magnetoelectroelastic body containing a moving crack under longitudinal shear are obtained. Fourier transforms and Fourier sine series are used to reduce the mixed boundary value problems of the crack, which is assumed to be permeable or impermeable, to dual integral equations, and then expressed in terms of Fredholm integral equations of the second kind. Results show that the stress intensity factors are influenced by the material constants, the geometry size ratio and the velocity of the crack, and the propagation of the crack possibly brings about branching phenomena.
\end{abstract}

(c) 2006 Elsevier Ltd. All rights reserved.

Keywords: Moving crack; Rectangular magnetoelectroelastic body; Longitudinal shear; Integral equation; Branch phenomena

\section{Introduction}

Composites made of piezoelectric/piezomagnetic materials exhibit magnetoelectric effects that are not present in single-phase piezoelectric or piezomagnetic materials. Consequently, they are extensively used as electric packaging, sensors and actuators, e.g., magnetic field probes, acoustic/ultrasonic devices, hydrophones, and transducers with the responsibility of electromagnetomechanical energy conversion [1,2]. Studies of the properties of piezoelectric/piezomagnetic composites have been carried out by numerous investigators in recent years [3-8]. When subjected to mechanical, magnetic and electrical loads in service, these materials can fail due to some defects, e.g., cracks, holes, etc. arising during their manufacturing process. Therefore, there is a growing interest among researchers in solving fracture mechanics problems in media possessing coupled piezoelectric, piezomagnetic and magnetoelectric effects, that is, magnetoelectroelastic effects. Recently, Song and Sih [9] investigated crack initiation behavior in a magnetoelectroelastic composite under in-plane deformation. Gao et al. [10] presented an exact treatment of crack problems in a magnetoelectroelastic solid subjected to far-field loadings. Wang and Mai [11] obtained the general two-dimensional solutions to the magnetoelectroelastic problem of a crack via the extended Stroh formalism. The same authors [12] also considered mode III

\footnotetext{
* Corresponding author. Tel./fax: +86 02287401572.

E-mail addresses: keqianghu@163.com, khu@stevens.edu.cn (K.-Q. Hu).
} 


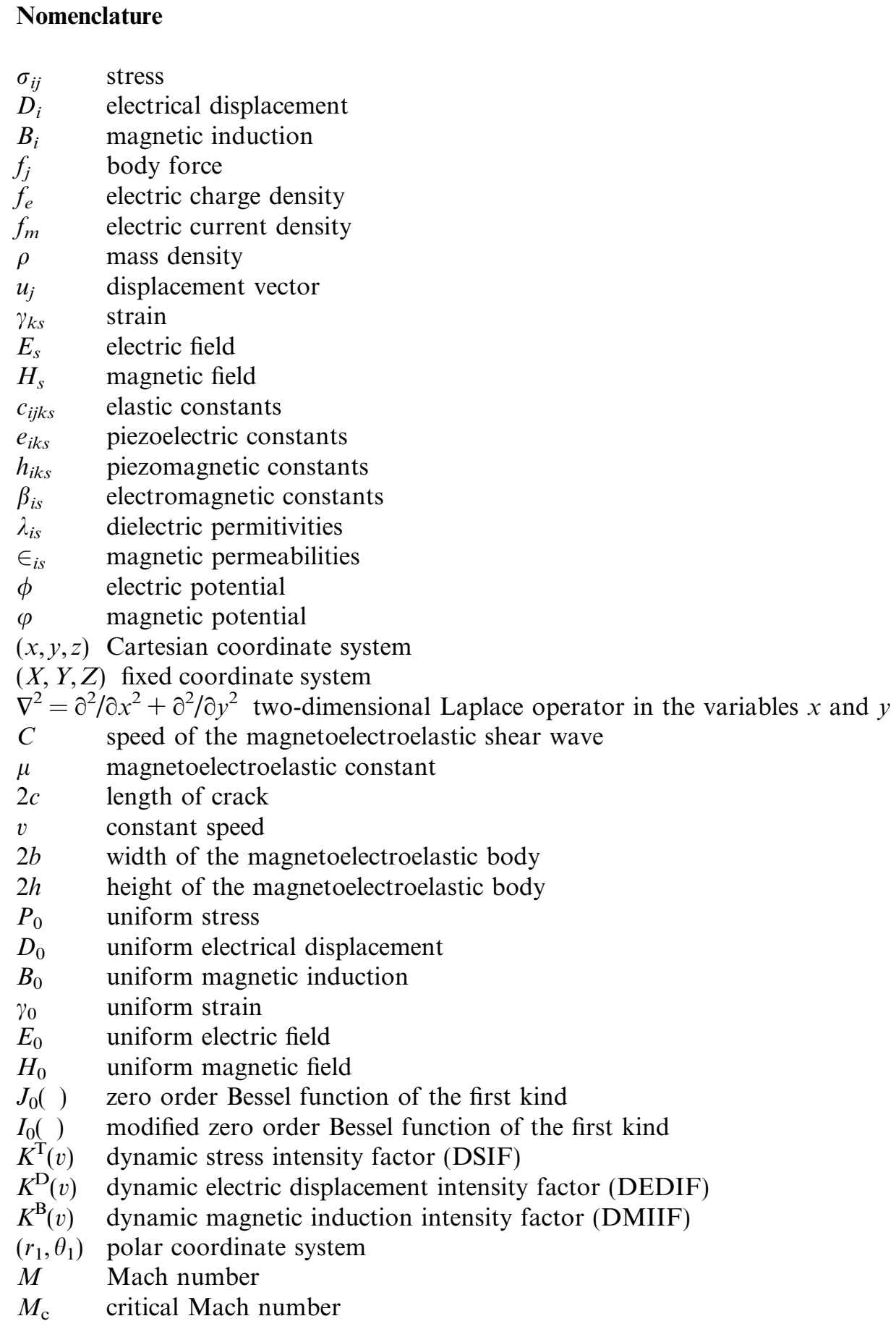

crack problems in an infinite magnetoelectroelastic medium using a complex variable technique. The dynamic behavior of two collinear interface cracks in magnetoelectroelastic materials under harmonic anti-plane shear wave loading was studied by Zhou et al. [13]. Qin [14] derived two-dimensional Green's functions of defective magnetoelectroelastic solids under thermal loading, which can be used to establish boundary formulations and to analyze relevant fracture problems. Li [15] performed transient analysis of a cracked magnetoelectroelastic 
medium under anti-plane mechanical and in-plane electric and magnetic impacts. The volume fraction effect of a magnetoelectroelastic composite on enhancement and impediment of crack growth was determined in [16]. The moving crack problem in an infinite magnetoelectroelastic material under longitudinal shear was studied by $\mathrm{Hu}$ and $\mathrm{Li}$ [17]. However, the moving crack problem in a rectangular magnetoelectroelastic body, rather than an infinite body, can more accurately reflect the reality as most engineering structures are in finite dimension. To the authors' knowledge, such problems have not yet been reported in the literature. That is the motivation of this work.

The objective of this paper is to seek the solution of the moving crack problem in a rectangular magnetoelectroelastic body under longitudinal shear. Fourier transforms and Fourier series are used to reduce the problem to the solution of dual integral equations. The solution of the dual integral equations is then expressed in terms of Fredholm integral equations of the second kind, and the series form solution of the integral equation is given. Explicit expressions of field variables and the field intensity factors are obtained, and results show that the corresponding field intensity factors are influenced by the material constants, the geometry size ratio and the velocity of the crack. It can be demonstrated that problems of an infinite magnetoelectroelastic material or of a magnetoelectroelastic strip containing a central crack under longitudinal shear are special cases of the general solution in this article.

\section{Basic equations for magnetoelectroelastic media}

We consider a linear magnetoelectroelastic solid and denote the rectangular coordinates of a point by $x_{j}$ $(j=1,2,3)$. The equilibrium equations are given as follows [5]:

$$
\sigma_{i j, i}+f_{j}=\rho \frac{\partial^{2} u_{j}}{\partial t^{2}}, \quad D_{i, i}-f_{e}=0, \quad B_{i, i}-f_{m}=0
$$

where $\sigma_{i j}, D_{i}$ and $B_{i}$ are components of stress, electrical displacement and magnetic induction, respectively; $f_{j}$, $f_{e}$ and $f_{m}$ are the body force, electric charge density, and electric current density, respectively; $\rho$ is the mass density of the magnetoelectroelastic material; $u_{j}$ is the displacement vector; a comma followed by $i$ $(i=1,2,3)$ denotes partial differentiation with respect to the coordinate $x_{i}$, and the usual summation convention over repeated indices is applied. The constitutive equations can be written as

$$
\begin{aligned}
& \sigma_{i j}=c_{i j k s} \gamma_{k s}-e_{s i j} E_{s}-h_{s i j} H_{s} \\
& D_{i}=e_{i k s} \gamma_{k s}+\lambda_{i s} E_{s}+\beta_{i s} H_{s} \\
& B_{i}=h_{i k s} \gamma_{k s}+\beta_{i s} E_{s}+\epsilon_{i s} H_{s}
\end{aligned}
$$

where $\gamma_{k s}, E_{s}$ and $H_{s}$ are components of strain, electric field and magnetic field, respectively; $c_{i j k s}, e_{i k s}, h_{i k s}$ and $\beta_{i s}$ are elastic, piezoelectric, piezomagnetic and electromagnetic constants, respectively; $\lambda_{i s}$ and $\in_{i s}$ are dielectric permitivities and magnetic permeabilities, respectively. The following reciprocal symmetries hold:

$$
\begin{aligned}
& c_{i j k s}=c_{j i k s}=c_{i j s k}=c_{k s i j}, \quad e_{s i j}=e_{s j i}, \\
& h_{s i j}=h_{s j i}, \quad \beta_{i j}=\beta_{j i}, \quad \lambda_{i j}=\lambda_{j i}, \quad \epsilon_{i j}=\epsilon_{j i}
\end{aligned}
$$

Gradient equations are

$$
\gamma_{i j}=\frac{1}{2}\left(u_{i, j}+u_{j, i}\right), \quad E_{i}=-\phi_{, i}, \quad H_{i}=-\varphi_{, i}
$$

where $\phi$ and $\varphi$ are electric potential and magnetic potential, respectively.

The governing equations simplify considerably if we consider only the out-of-plane displacement, the inplane electric fields and in-plane magnetic fields, i.e.,

$$
\begin{aligned}
& u_{1}=u_{2}=0, \quad u_{3}=w(X, Y) \\
& E_{1}=E_{X}(X, Y), \quad E_{2}=E_{Y}(X, Y), \quad E_{3}=0 \\
& H_{1}=H_{X}(X, Y), \quad H_{2}=H_{Y}(X, Y), \quad H_{3}=0
\end{aligned}
$$


The constitutive equations for anti-plane magnetoelectroelastic material take the form of [17]

$$
\left(\begin{array}{c}
\sigma_{Z Y} \\
D_{Y} \\
B_{Y}
\end{array}\right)=\left(\begin{array}{ccc}
c_{44} & e_{15} & h_{15} \\
e_{15} & -\lambda_{11} & -\beta_{11} \\
h_{15} & -\beta_{11} & -\epsilon_{11}
\end{array}\right)\left(\begin{array}{c}
\frac{\partial w}{\partial Y} \\
\frac{\partial \phi}{\partial Y} \\
\frac{\partial \varphi}{\partial Y}
\end{array}\right), \quad\left(\begin{array}{c}
\sigma_{Z X} \\
D_{X} \\
B_{X}
\end{array}\right)=\left(\begin{array}{ccc}
c_{44} & e_{15} & h_{15} \\
e_{15} & -\lambda_{11} & -\beta_{11} \\
h_{15} & -\beta_{11} & -\epsilon_{11}
\end{array}\right)\left(\begin{array}{c}
\frac{\partial w}{\partial X} \\
\frac{\partial \phi}{\partial X} \\
\frac{\partial \varphi}{\partial X}
\end{array}\right)
$$

In this case, if there is no body force, electric charge density and electric current density, the governing equations (1) simplify to

$$
\begin{aligned}
& c_{44} \nabla^{2} w+e_{15} \nabla^{2} \phi+h_{15} \nabla^{2} \varphi=\rho \frac{\partial^{2} w}{\partial t^{2}} \\
& e_{15} \nabla^{2} w-\lambda_{11} \nabla^{2} \phi-\beta_{15} \nabla^{2} \varphi=0 \\
& h_{15} \nabla^{2} w-\beta_{11} \nabla^{2} \phi-\epsilon_{11} \nabla^{2} \varphi=0
\end{aligned}
$$

where $\nabla^{2}=\partial^{2} / \partial x^{2}+\partial^{2} / \partial y^{2}$ is the two-dimensional Laplace operator in the variables $x$ and $y$.

Introducing two new functions $\Phi$ and $\Psi$ as

$$
\Phi=\phi+m_{1} \cdot w, \quad \Psi=\varphi+m_{2} \cdot w
$$

where

$$
m_{1}=\frac{\beta_{11} h_{15}-\epsilon_{11} e_{15}}{\lambda_{11} \epsilon_{11}-\beta_{11}^{2}}, \quad m_{2}=\frac{\beta_{11} e_{15}-\lambda_{11} h_{15}}{\lambda_{11} \epsilon_{11}-\beta_{11}^{2}}
$$

Eqs. (9) become

$$
\nabla^{2} w=\frac{1}{C^{2}} \frac{\partial^{2} w}{\partial t^{2}}, \quad \nabla^{2} \Phi=0, \quad \nabla^{2} \Psi=0
$$

where

$$
C=\sqrt{\frac{\mu}{\rho}}, \quad \mu=c_{44}+\frac{\epsilon_{11} e_{15}^{2}+\lambda_{11} h_{15}^{2}-2 \beta_{11} e_{15} h_{15}}{\lambda_{11} \epsilon_{11}-\beta_{11}^{2}}
$$

$C$ and $\mu$ are the speed of the magnetoelectroelastic shear wave and the magnetoelectroelastic constant, respectively.

\section{Problem statement and method of solution}

Consider a Griffith crack of finite length $2 c$ moving at constant speed $v$ in a rectangular magnetoelectroelastic body, which is subjected to mechanical, electrical and magnetic loadings as shown in Fig. 1. This type of crack is the so-called Yoffe-type moving crack which has been investigated by many researchers [17-26].

For convenience, let a Cartesian coordinate system $(x, y, z)$ be attached to the moving crack and when $t=0$ it coincides with the fixed coordinate system $(X, Y, Z)$. Since the problem is in a steady state, the Galilean transformation can be introduced, i.e.,

$$
x=X-v t, \quad y=Y, \quad z=Z
$$

With reference to the moving coordinates system, Eqs. (12) become independent of the time variable $t$ and may be rewritten as

$$
k^{2} \frac{\partial^{2} w(x, y)}{\partial x^{2}}+\frac{\partial^{2} w(x, y)}{\partial y^{2}}=0, \quad \nabla^{2} \Phi(x, y)=0, \quad \nabla^{2} \Psi(x, y)=0
$$

where

$$
k=\sqrt{1-(v / C)^{2}}
$$




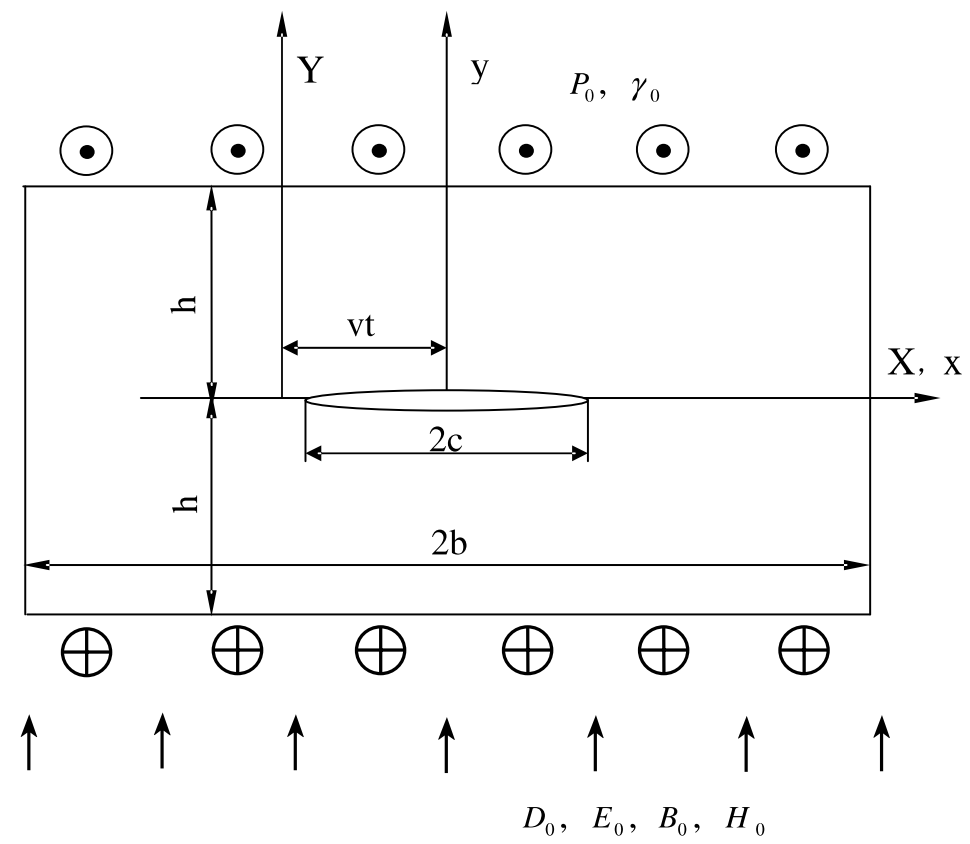

Fig. 1. Moving crack in a rectangular magnetoelectroelastic body under mechanical, electrical and magnetic loads.

The poled magnetoelectroelastic body with $z$ as the poling axis occupies the region $(-b \leqslant x \leqslant b$, $-h \leqslant y \leqslant h)$, and is thick enough in the $z$-direction to allow a state of anti-plane shear. A crack of finite length $2 c$ is situated along the plane $(-c<x<c, y=0)$. Due to the assumed symmetry in geometry and loading, it is sufficient to consider the problem for $0 \leqslant x \leqslant b, 0 \leqslant y \leqslant h$ only.

We consider eight possible cases of boundary conditions at the edges of the rectangular body:

Case 1: $\sigma_{y z}(x, h)=P_{0}, \quad D_{y}(x, h)=D_{0}, \quad B_{y}(x, h)=B_{0}$

Case 2: $\gamma_{y z}(x, h)=\gamma_{0}, \quad E_{y}(x, h)=E_{0}, \quad B_{y}(x, h)=B_{0}$

Case 3: $\sigma_{y z}(x, h)=P_{0}, \quad E_{y}(x, h)=E_{0}, \quad B_{y}(x, h)=B_{0}$

Case 4: $\gamma_{y z}(x, h)=\gamma_{0}, \quad D_{y}(x, h)=D_{0}, \quad B_{y}(x, h)=B_{0}$

Case 5: $\sigma_{y z}(x, h)=P_{0}, \quad D_{y}(x, h)=D_{0}, \quad H_{y}(x, h)=H_{0}$

Case 6: $\gamma_{y z}(x, h)=\gamma_{0}, \quad E_{y}(x, h)=E_{0}, \quad H_{y}(x, h)=H_{0}$

Case 7: $\sigma_{y z}(x, h)=P_{0}, \quad E_{y}(x, h)=E_{0}, \quad H_{y}(x, h)=H_{0}$

Case 8: $\gamma_{y z}(x, h)=\gamma_{0}, \quad D_{y}(x, h)=D_{0}, \quad H_{y}(x, h)=H_{0}$

The mechanical conditions are

$$
\begin{aligned}
& \sigma_{z y}(x, 0)=0 \quad(0 \leqslant x<c) \\
& w(x, 0)=u_{z}(x, 0)=0 \quad(c \leqslant x<b) \\
& \sigma_{z x}(b, y)=0 \quad(0 \leqslant y<h)
\end{aligned}
$$

The electrical and magnetic conditions for the permeable crack case can be expressed as [10,17,27]

$$
\begin{aligned}
& D_{y}\left(x, 0^{+}\right)=D_{y}\left(x, 0^{-}\right), \quad E_{x}\left(x, 0^{+}\right)=E_{x}\left(x, 0^{-}\right) \quad(0 \leqslant x<c) \\
& \phi(x, 0)=0 \quad(c \leqslant x<\infty) \\
& D_{x}(b, y)=0 \quad(0 \leqslant y<h)
\end{aligned}
$$




$$
\begin{aligned}
& B_{y}\left(x, 0^{+}\right)=B_{y}\left(x, 0^{-}\right), \quad H_{x}\left(x, 0^{+}\right)=H_{x}\left(x, 0^{-}\right) \quad(0 \leqslant x<c) \\
& \varphi(x, 0)=0 \quad(c \leqslant x<\infty) \\
& B_{x}(b, y)=0 \quad(0 \leqslant y<h)
\end{aligned}
$$

and the corresponding electrical and magnetic conditions for the impermeable crack are [12]

$$
\begin{array}{ll}
D_{y}(x, 0)=0 & (0 \leqslant x<c) \\
\phi(x, 0)=0 & (c \leqslant x<b) \\
B_{y}(x, 0)=0 & (0 \leqslant x<c) \\
\varphi(x, 0)=0 & (c \leqslant x<b)
\end{array}
$$

Fourier transforms and Fourier sine series $[28,29]$ are applied to Eqs. (15), and we obtain the results as

$$
\begin{aligned}
& w(x, y)=\frac{2}{\pi} \int_{0}^{\infty} A_{1}(\xi) \frac{\cosh [k s(h-y)]}{\cosh (k s h)} \cos (s x) \mathrm{d} s+\sum_{n=0}^{\infty} B_{1}(n) \cosh \left(\frac{\beta_{n} x}{k h}\right) \sin \left(\frac{\beta_{n} y}{h}\right)+a_{0} y \\
& \Phi(x, y)=\frac{2}{\pi} \int_{0}^{\infty} A_{2}(s) \frac{\cosh [s(h-y)]}{\cosh (s h)} \cos (s x) \mathrm{d} s+\sum_{n=0}^{\infty} B_{2}(n) \cosh \left(\frac{\beta_{n} x}{h}\right) \sin \left(\frac{\beta_{n} y}{h}\right)-b_{0} y \\
& \Psi(x, y)=\frac{2}{\pi} \int_{0}^{\infty} A_{3}(s) \frac{\cosh [s(h-y)]}{\cosh (s h)} \cos (s x) \mathrm{d} s+\sum_{n=0}^{\infty} B_{3}(n) \cosh \left(\frac{\beta_{n} x}{h}\right) \sin \left(\frac{\beta_{n} y}{h}\right)-c_{0} y
\end{aligned}
$$

where $A_{i}(\xi), B_{i}(\xi)(i=1,2,3)$ are the unknowns to be solved and $a_{0}, b_{0}, c_{0}$ are real constants which can be obtained by considering the edge loading conditions at $y=h$ as given in the appendix, and $\beta_{n}=(2 n+1) \pi / 2$. A simple calculation leads to the stress, electric displacement and magnetic induction expressions:

$$
\begin{aligned}
\sigma_{z x}= & -\frac{2}{\pi} \int_{0}^{\infty} s\left\{\mu A_{1}(s) \frac{\cosh [k s(h-y)]}{\cosh (k s h)}+\left[e_{15} A_{2}(s)+h_{15} A_{3}(s)\right] \frac{\cosh [s(h-y)]}{\cosh (s h)}\right\} \sin (s x) \mathrm{d} s \\
& +\sum_{n=0}^{\infty} \frac{\beta_{n}}{h}\left\{\frac{\mu}{k} B_{1}(n) \sinh \left(\frac{\beta_{n} x}{k h}\right)+\left[e_{15} B_{2}(n)+h_{15} B_{3}(n)\right] \sinh \left(\frac{\beta_{n} x}{h}\right)\right\} \sin \left(\frac{\beta_{n} y}{h}\right) \\
\sigma_{z y}= & -\frac{2}{\pi} \int_{0}^{\infty} s\left\{k \mu A_{1}(s) \frac{\sinh [k s(h-y)]}{\cosh (k s h)}+\left[e_{15} A_{2}(s)+h_{15} A_{3}(s)\right] \frac{\sinh [s(h-y)]}{\cosh (s h)}\right\} \cos (s x) \mathrm{d} s \\
& +\sum_{n=0}^{\infty} \frac{\beta_{n}}{h}\left\{\mu B_{1}(n) \cosh \left(\frac{\beta_{n} x}{k h}\right)+\left[e_{15} B_{2}(n)+h_{15} B_{3}(n)\right] \cosh \left(\frac{\beta_{n} x}{h}\right)\right\} \cos \left(\frac{\beta_{n} y}{h}\right)+d_{0} \\
D_{x}= & \frac{2}{\pi} \int_{0}^{\infty} s\left[\lambda_{11} A_{2}(s)+\beta_{11} A_{3}(s)\right] \frac{\cosh [s(h-y)]}{\cosh (s h)} \sin (s x) \mathrm{d} s \\
& -\sum_{n=0}^{\infty} \frac{\beta_{n}}{h}\left[\lambda_{11} B_{2}(n)+\beta_{11} B_{3}(n)\right] \sinh \left(\frac{\beta_{n} x}{h}\right) \sin \left(\frac{\beta_{n} y}{h}\right) \\
D_{y}= & \frac{2}{\pi} \int_{0}^{\infty} s\left[\lambda_{11} A_{2}(s)+\beta_{11} A_{3}(s)\right] \frac{\sinh [s(h-y)]}{\cosh (s h)} \cos (s x) \mathrm{d} s \\
& -\sum_{n=0}^{\infty} \frac{\beta_{n}}{h}\left[\lambda_{11} B_{2}(n)+\beta_{11} B_{3}(n)\right] \cosh \left(\frac{\beta_{n} x}{h}\right) \cos \left(\frac{\beta_{n} y}{h}\right)+\lambda_{11} b_{0}+\beta_{11} c_{0} \\
B_{x}= & \frac{2}{\pi} \int_{0}^{\infty} s\left[\beta_{11} A_{2}(s)+\epsilon_{11} A_{3}(s)\right] \frac{\cosh [s(h-y)]}{\cosh (s h)} \sin (s x) \mathrm{d} s \\
& -\sum_{n=0}^{\infty} \frac{\beta_{n}}{h}\left[\beta_{11} B_{2}(n)+\epsilon_{11} B_{3}(n)\right] \sinh \left(\frac{\beta_{n} x}{h}\right) \sin \left(\frac{\beta_{n} y}{h}\right) \\
B_{y}= & \frac{2}{\pi} \int_{0}^{\infty} s\left[\beta_{11} A_{2}(s)+\epsilon_{11} A_{3}(s)\right] \frac{\sinh [s(h-y)]}{\cosh (s h)} \cos (s x) \mathrm{d} s \\
& -\sum_{n=0}^{\infty} \frac{\beta_{n}}{h}\left[\beta_{11} B_{2}(n)+\epsilon_{11} B_{3}(n)\right] \cosh \left(\frac{\beta_{n} x}{h}\right) \cos \left(\frac{\beta_{n} y}{h}\right)+\beta_{11} b_{0}+\epsilon_{11} c_{0} \\
&
\end{aligned}
$$


where

$$
d_{0}=\mu a_{0}-e_{15} b_{0}-h_{15} c_{0}
$$

Substituting Eqs. (29), (31) and (33) into (19), (21) and (23), respectively, we obtain the following relations:

$$
\begin{aligned}
& B_{1}(n)=\frac{4}{\pi k h \sinh \left(\beta_{n} b / k h\right)} \int_{0}^{\infty} \frac{s}{s^{2}+\left(\beta_{n} / k h\right)^{2}} A_{1}(s) \sin (s b) \mathrm{d} s \\
& B_{i}(n)=\frac{4}{\pi h \sinh \left(\beta_{n} b / h\right)} \int_{0}^{\infty} \frac{s}{s^{2}+\left(\beta_{n} / h\right)^{2}} A_{i}(s) \sin (s b) \quad(i=2,3)
\end{aligned}
$$

\subsection{Permeable case solution}

Satisfaction of the three mixed boundary conditions (20) and (22) for a permeable crack leads to the result

$$
A_{i}(s)=m_{i-1} A_{1}(s) \quad(i=2,3)
$$

From the conditions (18), and using Eqs. (26), (30) and (36)-(37), we obtain the pair of dual integral equations of the following form:

$$
\begin{aligned}
& \int_{0}^{\infty} s\left\{\left[\frac{\mu}{c_{44}} \tanh (k s h)+\left(1-\frac{\mu}{c_{44}}\right) \tanh (s h)\right]\right\} A_{1}(s) \cos (s x) \mathrm{d} s \\
& -\frac{\pi}{2}\left\{\sum_{n=0}^{\infty} \frac{\beta_{n}}{k h} \cosh \left(\frac{\beta_{n} x}{k h}\right) \frac{4 \mu}{c_{44} \pi k h \sinh \left(\beta_{n} b / k h\right)} \int_{0}^{\infty} \frac{s A_{1}(s) \sin (s b)}{s^{2}+\left(\beta_{n} / k h\right)^{2}} \mathrm{~d} s\right. \\
& \left.\quad+\sum_{n=0}^{\infty} \frac{\beta_{n}}{k h} \cosh \left(\frac{\beta_{n} x}{h}\right) \frac{4\left(c_{44}-\mu\right)}{c_{44} \pi h \sinh \left(\beta_{n} b / h\right)} \int_{0}^{\infty} \frac{s A_{1}(s) \sin (s b)}{s^{2}+\left(\beta_{n} / h\right)^{2}} \mathrm{~d} s\right\}=\frac{\pi d_{0}}{2 c_{44} k} \quad(0 \leqslant x \leqslant c) \\
& \int_{0}^{\infty} A_{1}(s) \cos (s x) \mathrm{d}=0 \quad(c \leqslant x \leqslant b)
\end{aligned}
$$

To solve Eqs. (38), let $A_{1}(s)$ be expressed in the following integral representation by a new function $\Omega(t)$ :

$$
A_{1}(s)=\int_{0}^{c} t \Omega(t) J_{0}(s t) \mathrm{d} t
$$

where $J_{0}()$ represents the zero order Bessel function of the first kind.

Upon substituting Eq. (39) into Eqs. (38), and considering the identical expressions [30,31],

$$
\begin{aligned}
& \int_{0}^{\infty} J_{0}(s t) \cos (s x) \mathrm{d} s= \begin{cases}\frac{1}{\sqrt{t^{2}-x^{2}}} & |x|<t \\
0 & |x|>t\end{cases} \\
& \int_{0}^{\infty} \frac{s}{s^{2}+\left(\beta_{n} / h\right)^{2}} J_{0}(s t) \sin (s b) \mathrm{d} s=\frac{\pi}{2} \mathrm{e}^{-\left(\beta_{n} b / h\right)} I_{0}\left(\beta_{n} t / h\right)
\end{aligned}
$$

we find that the auxiliary function $\Omega(t)$ should satisfy the Fredholm integral equation of the second kind in the form

$$
\Omega(t)+\int_{0}^{c} \Omega(\eta)\left[K_{1}(t, \eta)+K_{2}(t, \eta)\right] d \eta=\frac{\pi d_{0}}{2 c_{44} k N}
$$

where

$$
\begin{aligned}
& K_{1}(t, \eta)=\frac{\eta}{N} \int_{0}^{\infty} s\left\{\frac{\mu}{c_{44}}[\tanh (k s h)-1]+\left(1-\frac{\mu}{c_{44}}\right) \frac{[\tanh (s h)-1]}{k}\right\} J_{0}(s t) J_{0}(s \eta) \mathrm{d} s \\
& K_{2}(t, \eta)=\frac{-\eta \pi \beta_{n}}{N k h^{2}} \sum_{n=0}^{\infty}\left\{\frac{\mu}{c_{44}} \frac{\mathrm{e}^{-\left(\beta_{n} b / k h\right)}}{k \sinh \left(\beta_{n} b / k h\right)} I_{0}\left(\beta_{n} \eta / k h\right) I_{0}\left(\beta_{n} t / k h\right)\right. \\
& \left.+\left(1-\frac{\mu}{c_{44}}\right) \frac{\mathrm{e}^{-\left(\beta_{n} b / h\right)}}{\sinh \left(\beta_{n} b / h\right)} I_{0}\left(\beta_{n} \eta / h\right) I_{0}\left(\beta_{n} t / h\right)\right\} \\
& N=\frac{c_{44}+(k-1) \mu}{c_{44} k}
\end{aligned}
$$

and $I_{0}($ ) is the modified zero order Bessel function of the first kind. 
Employing the following variables and functions to normalize the Fredholm integral equation of the second kind (42):

$$
\begin{aligned}
& \eta=c H, \quad s=S / c, \quad t=c E \\
& \Omega(t)=\frac{\pi d_{0}}{2 c_{44} k N} \Xi(E), \quad \Omega(\eta)=\frac{\pi d_{0}}{2 c_{44} k N} \Xi(H)
\end{aligned}
$$

we obtain

$$
\Xi(E)+\int_{0}^{1} \Xi(H)\left[L_{1}(E, H)-L_{2}(E, H)\right] \mathrm{d} H=1
$$

where

$$
\begin{aligned}
L_{1}(E, H)= & \frac{H}{N} \int_{0}^{\infty} S\left\{\frac{\mu}{c_{44}}\left[\tanh \left(\frac{k S h}{c}\right)-1\right]+\left(1-\frac{\mu}{c_{44}}\right) \tanh \left(\frac{S h}{c}\right)-1\right\} J_{0}(S E) J_{0}(S H) \mathrm{d} S \\
L_{2}(E, H)= & \frac{H \pi \beta_{n} c^{2}}{N k h^{2}} \sum_{n=0}^{\infty}\left\{\frac{\mu}{c_{44}} \frac{\mathrm{e}^{-\left(\beta_{n} b / k h\right)}}{k \sinh \left(\beta_{n} b / k h\right)} I_{0}\left(\beta_{n} H c / k h\right) I_{0}\left(\beta_{n} E c / k h\right)\right. \\
& \left.+\left(1-\frac{\mu}{c_{44}}\right) \frac{\mathrm{e}^{-\left(\beta_{n} b / h\right)}}{\sinh \left(\beta_{n} b / h\right)} I_{0}\left(\beta_{n} H c / h\right) I_{0}\left(\beta_{n} E c / h\right)\right\}
\end{aligned}
$$

Substitution of Eqs. (46)-(47) into Eq. (39) gives

$$
A_{1}(s)=\frac{\pi d_{0} c^{2}}{2 c_{44} k N} \int_{0}^{1} E \Xi(E) J_{0}(s c E) \mathrm{d} E
$$

\subsection{Impermeable case solution}

Using the mixed boundary conditions (18)-(19) and (24)-(25), we obtain the following simultaneous dual integral equations:

$$
\begin{aligned}
& \int_{0}^{\infty} s A_{1}(s) \tanh (k s h) \cos (s x) \mathrm{d} s-\frac{\pi}{2 k} \sum_{n=0}^{\infty} \frac{\beta_{n}}{h} B_{1}(n) \cosh \left(\beta_{n} x / k h\right)=\frac{\pi T_{1}}{2} \quad(0 \leqslant x \leqslant c) \\
& \int_{0}^{\infty} A_{1}(s) \cos (s x) \mathrm{d} s=0 \quad(c \leqslant x \leqslant b) \\
& \int_{0}^{\infty} s A_{i}(s) \tanh (s h) \cos (s x) \mathrm{d} s-\frac{\pi}{2} \sum_{n=0}^{\infty} \frac{\beta_{n}}{h} B_{i}(n) \cosh \left(\beta_{n} x / h\right)=\frac{\pi T_{i}}{2} \quad(i=2,3,0 \leqslant x \leqslant c) \\
& \int_{0}^{\infty} A_{i}(s) \cos (s x) \mathrm{d} s=0 \quad(c \leqslant x \leqslant b)
\end{aligned}
$$

where

$$
T_{1}=a_{0} / k, \quad T_{2}=-b_{0}, \quad T_{3}=-c_{0}
$$

It is easily seen from Eqs. (53) and (54) that

$$
A_{3}(s)=\frac{c_{0}}{b_{0}} A_{2}(s)
$$

Substituting Eq. (55) into Eqs. (52)-(53) and letting

$$
A_{i}(s)=\frac{\pi T_{i} c^{2}}{2} \int_{0}^{1} E \Pi_{i}(E) J_{0}(s c E) \mathrm{d} E \quad(i=1,2)
$$


we find that the auxiliary functions $\Pi_{i}(E)(i=1,2)$ are governed by the Fredholm integral equations in the form,

$$
\Pi_{i}(E)+\int_{0}^{1} \Pi_{i}(H)\left[F_{i 1}(E, H)-F_{i 2}(E, H)\right] \mathrm{d} H=1 \quad(i=1,2)
$$

where

$$
\begin{aligned}
& F_{i 1}(E, H)=H \int_{0}^{\infty} S\left[\tanh \left(k_{i} S h / c\right)-1\right] J_{0}(S E) J_{0}(S H) \mathrm{d} S \\
& F_{i 2}(E, H)=\frac{H \pi \beta_{n} c^{2}}{\left(k_{i} h\right)^{2}} \sum_{n=0}^{\infty} \frac{\mathrm{e}^{-\left(\beta_{n} b / k_{i} h\right)}}{\sinh \left(\beta_{n} b / k_{i} h\right)} I_{0}\left(\beta_{n} H c / k_{i} h\right) I_{0}\left(\beta_{n} E c / k_{i} h\right)
\end{aligned}
$$

and

$$
k_{i}= \begin{cases}k & (i=1) \\ 1 & (i=2)\end{cases}
$$

\section{Field intensity factors}

The singular parts of the stresses, the electric displacements and the magnetic inductions near the right crack tip in the permeable boundary case are

$$
\begin{aligned}
\sigma_{z y} & =\frac{K^{\mathrm{T}}(v)}{\sqrt{2 \pi r_{1}}}\left[(1+q) \sqrt{\frac{r_{1}}{\tilde{r}_{1}}} \cos \left(\tilde{\theta}_{1} / 2\right)-q \cos \left(\theta_{1} / 2\right)\right] \\
\sigma_{z x} & =-\frac{K^{\mathrm{T}}(v)}{\sqrt{2 \pi r_{1}}}\left[\frac{(1+q)}{k} \sqrt{\frac{r_{1}}{\tilde{r}_{1}}} \sin \left(\tilde{\theta}_{1} / 2\right)-q \sin \left(\theta_{1} / 2\right)\right] \\
D_{y} & =\frac{K^{\mathrm{D}}(v)}{\sqrt{2 \pi r_{1}}} \cos \left(\frac{\theta_{1}}{2}\right), \quad D_{x}=-\frac{K^{\mathrm{D}}(v)}{\sqrt{2 \pi r_{1}}} \sin \left(\frac{\theta_{1}}{2}\right) \\
B_{y} & =\frac{K^{\mathrm{B}}(v)}{\sqrt{2 \pi r_{1}}} \cos \left(\frac{\theta_{1}}{2}\right), \quad B_{x}=-\frac{K^{\mathrm{B}}(v)}{\sqrt{2 \pi r}} \sin \left(\frac{\theta_{1}}{2}\right)
\end{aligned}
$$

where

$$
\begin{aligned}
& \kappa=k+(k-1) \alpha, \quad \alpha=\mu / c_{44}-1=\frac{\in_{11} e_{15}^{2}+\lambda_{11} h_{15}^{2}-2 \beta_{11} e_{15} h_{15}}{c_{44}\left(\lambda_{11} \epsilon_{11}-\beta_{11}^{2}\right)} \\
& q=\frac{\alpha}{\kappa}=\frac{\epsilon_{11} e_{15}^{2}+\lambda_{11} h_{15}^{2}-2 \beta_{11} e_{15} h_{15}}{c_{44}\left(\lambda_{11} \in_{11}-\beta_{11}^{2}\right) k+\left(\epsilon_{11} e_{15}^{2}+\lambda_{11} h_{15}^{2}-2 \beta_{11} e_{15} h_{15}\right)(k-1)}
\end{aligned}
$$

and $r_{1}, \tilde{r}_{1}, \theta_{1}$ and $\tilde{\theta}_{1}$ are defined respectively as

$$
\begin{aligned}
& r_{1}=\sqrt{(x-c)^{2}+y^{2}}, \quad \theta_{1}=\arctan \left(\frac{y}{x-c}\right) \\
& \tilde{r}_{1}=\sqrt{(x-c)^{2}+(k y)^{2}}, \quad \tilde{\theta}_{1}=\arctan \left(\frac{k y}{x-c}\right)
\end{aligned}
$$

$K^{\mathrm{T}}(v), K^{\mathrm{D}}(v)$ and $K^{\mathrm{B}}(v)$ are the dynamic stress intensity factor (DSIF), the dynamic electric displacement intensity factor (DEDIF), and the dynamic magnetic induction intensity factor (DMIIF), respectively. These field intensity factors can be defined as

$$
\begin{aligned}
& K^{\mathrm{T}}(v)=\lim _{x \rightarrow c^{+}} \sqrt{2 \pi(x-c)} \sigma_{z y}(x, 0)=d_{0} \sqrt{\pi c} \Xi(1) \\
& K^{\mathrm{D}}(v)=\lim _{x \rightarrow c^{+}} \sqrt{2 \pi(x-c)} D_{y}(x, 0)=\frac{e_{15}}{c_{44} \kappa} K^{\mathrm{T}}(v)=\frac{e_{15} d_{0}}{c_{44} \kappa} \sqrt{\pi c} \Xi(1) \\
& K^{\mathrm{B}}(v)=\lim _{x \rightarrow c^{+}} \sqrt{2 \pi(x-c)} B_{y}(x, 0)=\frac{h_{15}}{c_{44} \kappa} K^{\mathrm{T}}(v)=\frac{h_{15} d_{0}}{c_{44} \kappa} \sqrt{\pi c} \Xi(1)
\end{aligned}
$$


For this particular problem, the stresses, electric displacements and magnetic inductions at the crack tip show inverse square root singularities. It is clear that the DSIF, DEDIF and DMIIF under the permeable crack condition are dependent on the velocity of the moving crack, the geometry size of the rectangular body, the load conditions and the material constants.

Using the polar coordinate system $\left(r_{1}, \theta_{1}\right)$ defined near by the crack tip, the DSIF along the orientation $\theta_{1}$ can be obtained as

$$
K^{\mathrm{T}}\left(v, \theta_{1}\right)=d_{0} \sqrt{\pi c} F_{1}\left(v, \theta_{1}\right)
$$

where

$$
\begin{aligned}
& F_{1}\left(v, \theta_{1}\right)=\left\{(1+q) \Omega\left(\theta_{1}\right)\left[\cos \left(\theta_{1}\right) \cos \left(\frac{\tilde{\theta}_{1}}{2}\right)+\frac{1}{k} \sin \left(\theta_{1}\right) \sin \left(\frac{\tilde{\theta}_{1}}{2}\right)\right]-q \cos \left(\frac{\theta_{1}}{2}\right)\right\} \Xi(1) \\
& \Omega\left(\theta_{1}\right)=\sqrt{\frac{r_{1}}{\tilde{r}_{1}}}=\frac{1}{4 \sqrt{4} k^{2}+\left(1-k^{2}\right) \cos ^{2}\left(\theta_{1}\right)}, \quad \tan \left(\tilde{\theta}_{1}\right)=k \tan \left(\theta_{1}\right)
\end{aligned}
$$

To illustrate the influence of the velocity of the moving crack on the DSIF, a Mach number as the ratio of the velocity to the magnetoelectroelastic shear wave speed, $M=v / C$, is introduced. It can be observed from Eqs. (64) and (68)-(69) that the magnitudes of $K^{\mathrm{D}}(v)$ and $K^{\mathrm{B}}(v)$, in the case of permeable condition will become infinity when

$$
M=M_{d}=\frac{\sqrt{c_{44}\left(\lambda_{11} \epsilon_{11}-\beta_{11}^{2}\right)\left[c_{44}\left(\lambda_{11} \epsilon_{11}-\beta_{11}^{2}\right)+2\left(\epsilon_{11} e_{15}^{2}+\lambda_{11} h_{15}^{2}-2 \beta_{11} e_{15} h_{15}\right)\right]}}{c_{44}\left(\lambda_{11} \epsilon_{11}-\beta_{11}^{2}\right)+\epsilon_{11} e_{15}^{2}+\lambda_{11} h_{15}^{2}-2 \beta_{11} e_{15} h_{15}}
$$

In the case of $\beta_{11}=0$ and $h_{15}=0$, our results are exactly reduced to the corresponding piezoelectric solutions, and are in agreement with the results of Kwon and Lee [23]. This shows that our solutions are correct and universal.

For the impermeable crack case, the asymptotic fields in the neighborhood of the propagating crack tip can be written as

$$
\begin{aligned}
\sigma_{z y} & =\frac{K^{\mathrm{IT}}(v)}{\sqrt{2 \pi r_{1}}}\left[\Re \sqrt{\frac{r_{1}}{\tilde{r}_{1}}} \cos \left(\tilde{\theta}_{1} / 2\right)+(1-\mathfrak{R}) \cos \left(\theta_{1} / 2\right)\right] \\
\sigma_{z x} & =-\frac{K^{\mathrm{IT}}(v)}{\sqrt{2 \pi r_{1}}}\left[\frac{\mathfrak{R}}{k} \sqrt{\frac{r_{1}}{\tilde{r}_{1}}} \sin \left(\tilde{\theta}_{1} / 2\right)+(1-\mathfrak{R}) \sin \left(\theta_{1} / 2\right)\right] \\
D_{y} & =\frac{K^{\mathrm{ID}}(v)}{\sqrt{2 \pi \tilde{r}_{1}}} \cos \left(\frac{\tilde{\theta}_{1}}{2}\right), \quad D_{x}=-\frac{K^{\mathrm{ID}}(v)}{\sqrt{2 \pi \tilde{r}_{1}}} \sin \left(\frac{\tilde{\theta}_{1}}{2}\right) \\
B_{y} & =\frac{K^{\mathrm{IB}}(v)}{\sqrt{2 \pi \tilde{r}_{1}}} \cos \left(\frac{\tilde{\theta}_{1}}{2}\right), \quad B_{x}=-\frac{K^{\mathrm{IB}}(v)}{\sqrt{2 \pi \tilde{r}_{1}}} \sin \left(\frac{\tilde{\theta}_{1}}{2}\right)
\end{aligned}
$$

where $K^{\mathrm{TT}}(v), K^{\mathrm{ID}}(v)$ and $K^{\mathrm{IB}}(v)$ are the DSIF, the DEDIF and DMIIF, respectively; these field intensity factors can be defined as

$$
\begin{aligned}
& K^{\mathrm{IT}}(v)=\left[\mu a_{0} \Pi_{1}(1)-\left(e_{15} b_{0}+h_{15} c_{0}\right) \Pi_{2}(1)\right] \sqrt{\pi c} \\
& K^{\mathrm{ID}}(v)=\left(\lambda_{11} b_{0}+\beta_{11} c_{0}\right) \Pi_{2}(1) \sqrt{\pi c} \\
& K^{\mathrm{IB}}(v)=\left(\beta_{11} b_{0}+\epsilon_{11} c_{0}\right) \Pi_{2}(1) \sqrt{\pi c}
\end{aligned}
$$

and $\mathfrak{R}$ are given by

$$
\mathfrak{R}=\frac{\mu a_{0} \Pi_{1}(1)}{\mu a_{0} \Pi_{1}(1)-\left(e_{15} b_{0}+h_{15} c_{0}\right) \Pi_{2}(1)}
$$


The DSIF for the impermeable crack along the orientation $\theta_{1}$ can be obtained as

$$
K^{\mathrm{IT}}\left(v, \theta_{1}\right)=\left[\mu a_{0}-\left(e_{15} b_{0}+h_{15} c_{0}\right)\right] \sqrt{\pi c} F_{2}\left(v, \theta_{1}\right)
$$

where

$$
\begin{aligned}
& F_{2}\left(v, \theta_{1}\right)=p \Pi_{1}(1) \Omega\left(\theta_{1}\right)\left[\cos \left(\theta_{1}\right) \cos \left(\frac{\tilde{\theta}_{1}}{2}\right)+\frac{1}{k} \sin \left(\theta_{1}\right) \sin \left(\frac{\tilde{\theta}_{1}}{2}\right)\right]+(1-p) \Pi_{2}(1) \cos \left(\frac{\theta_{1}}{2}\right) \\
& p=\frac{\mu a_{0}}{\mu a_{0}-\left(e_{15} b_{0}+h_{15} c_{0}\right)}
\end{aligned}
$$

It can be seen from Eqs. (71) and (81) that the functions $F_{1}\left(v, \theta_{1}\right)$ and $F_{2}\left(v, \theta_{1}\right)$ are independent of the crack length $2 c$, and the crack length does not affect the distribution of the DSIF on the circumference. Therefore, analyzing the functions $F_{i}\left(v, \theta_{1}\right)(i=1,2)$ would provide a good model to understand crack propagation orientation.

For this particular problem, the stresses, electric displacements and magnetic inductions at the crack tip show inverse square root singularities. It is clear that the DSIF, DEDIF and DMIIF under the permeable or impermeable crack conditions are dependent on the velocity of the moving crack, the geometry of the rectangular body, the load conditions and the material constants.

\section{Numerical results and discussion}

From expressions (67)-(69) and (71) we know that the determination of the dynamic field intensity factors for the permeable crack case must require the solution of the function $\Xi(1)=\left.\Xi(E)\right|_{E=1}$. The solution of the Fredholm integral equation (48) may be given as

$$
\Xi(E)=\sum_{n=0}^{\infty} \Xi_{n}(E)
$$

in which

$$
\Xi_{0}(E)=1, \quad \Xi_{n+1}(E)=\int_{0}^{1}\left(L_{2}-L_{1}\right) \Xi_{n}(H) \mathrm{d} H, \quad n=0,1,2, \ldots
$$

Following the procedure given in [28] and using the following identities

$$
\begin{aligned}
& J_{0}(S E) J_{0}(S H)=1-\frac{H^{2}+E^{2}}{4} S^{2}+\frac{H^{4}+4 H^{2} E^{2}+E^{4}}{64} S^{4}-\cdots \\
& I_{0}\left(\frac{\beta_{n} H c}{h}\right) I_{0}\left(\frac{\beta_{n} E c}{h}\right)=1+\frac{H^{2}+E^{2}}{4}\left(\frac{\beta_{n} c}{h}\right)^{2}+\frac{H^{4}+4 H^{2} E^{2}+E^{4}}{64}\left(\frac{\beta_{n} c}{h}\right)^{4}+\cdots \\
& \tanh (k S h / c)=1-2 \mathrm{e}^{-2 k S h / c}+2 \mathrm{e}^{-4 k S h / c}-2 \mathrm{e}^{-6 k S h / c}+\cdots
\end{aligned}
$$

we can obtain the solution of the function $\Xi(1)$ in series form for the higher values of the ratio $h / c$, i.e.,

$$
\begin{aligned}
\Xi(1)= & +\frac{1}{N}\left\{\frac{\pi^{2}}{48 k}\left(\frac{c}{h}\right)^{2}\left[1+\frac{\mu}{c_{44}}\left(\frac{1}{k}-1\right)\right]-\frac{7 \pi^{4}}{5120 k}\left(\frac{c}{h}\right)^{2}\left[1+\frac{\mu}{c_{44}}\left(\frac{1}{k^{3}}-1\right)\right]+\sum_{n=1,3 \ldots}^{\infty} \frac{\mathrm{e}^{-n \pi b / 2 k h}}{\sinh \left(\frac{n \pi b}{2 k h}\right)}\right. \\
& \left.\times \frac{\mu}{c_{44}}\left[\frac{n \pi^{2}}{4}\left(\frac{c}{k h}\right)^{2}+\frac{3 n^{3} \pi^{4}}{128}\left(\frac{c}{k h}\right)^{4}\right]+\frac{\mathrm{e}^{-n \pi b / 2 h}}{\sinh \left(\frac{n \pi b}{2 h}\right)}\left(1-\frac{\mu}{c_{44}}\right)\left[\frac{n \pi^{2}}{4 k}\left(\frac{c}{h}\right)^{2}+\frac{3 n^{3} \pi^{4}}{128 k}\left(\frac{c}{h}\right)^{4}\right]\right\} \\
& +\frac{1}{N^{2}}\left\{\sum_{n=1,3 \ldots}^{\infty} \frac{n \pi^{4}}{96 k^{2}}\left(\frac{c}{h}\right)^{4} \Delta_{n}\left[1+\frac{\mu}{c_{44}}\left(\frac{1}{k}-1\right)\right]+\sum_{m=1,3 \ldots}^{\infty} \sum_{n=1,3}^{\infty} \frac{m n \pi^{4}}{16 k^{2}}\left(\frac{c}{h}\right)^{4} \Delta_{n} \Delta_{m} .\right. \\
& \left.+\frac{\pi^{4}}{2304 k^{2}}\left(\frac{c}{h}\right)^{4}\left[1+\frac{\mu}{c_{44}}\left(\frac{1}{k}-1\right)\right]^{2}\right\}+\mathrm{O}\left(\frac{c}{h}\right)^{6}
\end{aligned}
$$


where

$$
\Delta_{i}=\frac{\mathrm{e}^{-\mathrm{i} \pi b / 2 k h}}{\sinh \left(\frac{i \pi b}{2 k h}\right)} \frac{\mu}{c_{44} k}+\frac{\mathrm{e}^{-\mathrm{i} \pi b / 2 h}}{\sinh \left(\frac{i \pi b}{2 h}\right)}\left(1-\frac{\mu}{c_{44}}\right) \quad(i=m, n)
$$

The solution of a magnetoelectroelastic strip with a central crack parallel to the strip edges may be derived from Eqs. (48)-(50) by considering $b \rightarrow \infty$ and noticing

$$
\lim _{b \rightarrow \infty} \frac{\mathrm{e}^{-n \pi b / 2 k h}}{\sinh (n \pi b / 2 k h)}=0
$$

the function $\Xi(E)$ should satisfy the Fredholm integral equation of the second kind in the form,

$$
\Xi(E)+\int_{0}^{1} \Xi(H) L_{1}(E, H) \mathrm{d} H=1
$$

where the function $L_{2}(E, H)$ in the Fredholm integral equation (48) automatically vanishes, and the function $\Xi(1)$ can be found in series form as

$$
\begin{aligned}
\Xi(1)= & 1+\frac{1}{N}\left\{\frac{\pi^{2}}{48 k}\left(\frac{c}{h}\right)^{2}\left[1+\frac{\mu}{c_{44}}\left(\frac{1}{k}-1\right)\right]-\frac{7 \pi^{4}}{5120 k}\left(\frac{c}{h}\right)^{2}\left[1+\frac{\mu}{c_{44}}\left(\frac{1}{k^{3}}-1\right)\right]\right. \\
& \left.+\frac{\pi^{4}}{2304 k^{2}}\left(\frac{c}{h}\right)^{4}\left[1+\frac{\mu}{c_{44}}\left(\frac{1}{k}-1\right)\right]^{2}\right\}+\mathrm{O}\left(\frac{c}{h}\right)^{6}
\end{aligned}
$$

Letting $h \rightarrow \infty$, and noticing

$$
\begin{aligned}
& \lim _{h \rightarrow \infty} \tanh (k S h / c)=1, \quad \lim _{h \rightarrow \infty} \tanh (S h / c)=1 \\
& \lim _{h \rightarrow \infty}(\pi c / h)=\mathrm{d} S, \quad \frac{n \pi c}{2 h}=S
\end{aligned}
$$

we may obtain the solution of a strip containing a central crack perpendicular to its edges. In this case, the corresponding Fredholm integral equation becomes

$$
\Xi(E)+\int_{0}^{1} \Xi(H) L_{3}(E, H) \mathrm{d} H=1
$$

where

$$
\begin{aligned}
L_{3}(E, H)= & \frac{H}{N k}\left\{\frac{\mu}{c_{44} k} \int_{0}^{\infty} S[1-c t h(S b / k c)] I_{0}(S H / k) I_{0}(S E / k) \mathrm{d} S\right. \\
& \left.+\left(1-\frac{\mu}{c_{44}}\right) \int_{0}^{\infty} S[1-c t h(S b / c)] I_{0}(S H) I_{0}(S E) \mathrm{d} S\right\}
\end{aligned}
$$

The function $\Xi(1)$ in this case takes the series form

$$
\begin{aligned}
\Xi(1)= & 1+\frac{1}{N}\left[\frac{\mu}{c_{44}}+\left(1-\frac{\mu}{c_{44}}\right) \frac{1}{k}\right]\left[\frac{\pi^{2}}{24}\left(\frac{c}{b}\right)^{2}+\frac{\pi^{4}}{640}\left(\frac{c}{b}\right)^{4}\right]+\frac{1}{N^{2}}\left[\frac{\mu}{c_{44}}+\left(1-\frac{\mu}{c_{44}}\right) \frac{1}{k}\right]^{2} \frac{\pi^{4}}{576}\left(\frac{c}{b}\right)^{4} \\
& +\mathrm{O}\left(\frac{c}{b}\right)^{6} \quad\left(\frac{c}{b} \ll 1\right)
\end{aligned}
$$

We can obtain the solution for a constant crack moving in an infinite piezomagnetoelectric material if we let $h \rightarrow \infty$ and $b \rightarrow \infty$, with $\Xi(1) \equiv 1$; which agrees with the result in [17].

Letting $v=0$ or $k=1$, the static solution of a finite rectangular magnetoelectroelastic body with a central crack under longitudinal shear may be easily deduced from (48)-(50), (85) and (86). The corresponding Fredholm integral equation is

$$
\Xi(E)+\int_{0}^{1} \Xi(H)\left[L_{4}(E, H)-L_{5}(E, H)\right] \mathrm{d} H=1
$$


where

$$
\begin{aligned}
& L_{4}(E, H)=H \int_{0}^{\infty} S[\tanh (S h / c)-1] J_{0}(S E) J_{0}(S H) \mathrm{d} S \\
& L_{5}(E, H)=H \sum_{n=0}^{\infty} \pi \beta_{n}\left(\frac{c}{h}\right)^{2} \frac{\mathrm{e}^{-\left(\beta_{n} b / h\right)}}{\sinh \left(\beta_{n} b / h\right)} I_{0}\left(\beta_{n} H c / h\right) I_{0}\left(\beta_{n} E c / h\right)
\end{aligned}
$$

and the function in series form is

$$
\begin{aligned}
\Xi(1)= & 1+\left(\frac{\pi^{2}}{48}-\frac{7 \pi^{4}}{5120}\right)\left(\frac{c}{h}\right)^{2}+\frac{\pi^{4}}{2304}\left(\frac{c}{h}\right)^{4} \\
& +\sum_{n=1,3 \ldots}^{\infty} \frac{n \pi^{4}}{96} \frac{\mathrm{e}^{-n \pi b / 2 h}}{\sinh \left(\frac{n \pi b}{2 h}\right)}\left(\frac{c}{h}\right)^{4}+\sum_{n=1,3 \ldots}^{\infty} \frac{\mathrm{e}^{-n \pi b / 2 h}}{\sinh \left(\frac{n \pi b}{2 h}\right)}\left[\frac{n \pi^{2}}{4}\left(\frac{c}{h}\right)^{2}+\frac{3 n^{3} \pi^{4}}{128}\left(\frac{c}{h}\right)^{4}\right] \\
& +\sum_{m=1,3 \ldots}^{\infty} \sum_{n=1,3}^{\infty} \frac{m n \pi^{4}}{16}\left(\frac{c}{h}\right)^{4} \frac{\mathrm{e}^{-(m+n) \pi b / 2 h}}{\sinh \left(\frac{n \pi b}{2 h}\right) \sinh \left(\frac{m \pi b}{2 h}\right)}+\mathrm{O}\left(\frac{c}{h}\right)^{6}
\end{aligned}
$$

The static solution of an infinitely long magnetoelectroelastic strip containing a central crack parallel to the strip edges [32] can be derived from Eqs. (48)-(50) by considering $b \rightarrow \infty$, and letting $v=0$.

In this case, the function $\Xi(E)$ is governed by

$$
\Xi(E)+\int_{0}^{1} \Xi(H) K(E, H) \mathrm{d} H=1
$$

with

$$
K(E, H)=H \int_{0}^{\infty} S[\tanh (S h / c)-1] J_{0}(S E) J_{0}(S H) \mathrm{d} S
$$

Determination of the dynamic field intensity factors for the impermeable crack case requires solution of the functions $\Pi_{i}(E)(i=1,2)$. The solution of the Fredholm integral equations (57) may be given in series form for higher ratio $h / c$ as

$$
\begin{aligned}
\Pi_{i}(1)= & 1+\frac{\pi^{2}}{48}\left(\frac{c}{k_{i} h}\right)^{2}-\frac{7 \pi^{4}}{5120}\left(\frac{c}{k_{i} h}\right)^{2} \\
& +\sum_{n=1,3 \ldots}^{\infty} \frac{\mathrm{e}^{-n \pi b / 2 k_{i} h}}{\sinh \left(\frac{n \pi b}{2 k_{i} h}\right)}\left[\frac{n \pi^{2}}{4}\left(\frac{c}{k_{i} h}\right)^{2}+\frac{3 n^{3} \pi^{4}}{128}\left(\frac{c}{k_{i} h}\right)^{4}+\frac{n \pi^{4}}{96}\left(\frac{c}{k_{i} h}\right)^{4}\right] \\
& +\sum_{m=1,3 \ldots}^{\infty} \sum_{n=1,3}^{\infty} \frac{\mathrm{e}^{-(m+n) \pi b / 2 k_{i} h}}{\sinh \left(\frac{m \pi b}{2 k_{i} h}\right) \sinh \left(\frac{n \pi b}{2 k_{i} h}\right)} \frac{m n \pi^{4}}{16}\left(\frac{c}{k_{i} h}\right)^{4}+\mathrm{O}\left(\frac{c}{h}\right)^{6}
\end{aligned}
$$

where $k_{i}(i=1,2)$ was defined in Eq. (60).

By considering $b \rightarrow \infty$ in Eqs. (57)-(59), we can obtain the solution for a central crack in a magnetoelectroelastic strip under impermeable conditions, and the auxiliary functions $\Pi_{i}(E)(i=1,2)$ satisfy the Fredholm integral equations in the form

$$
\Pi_{i}(E)+\int_{0}^{1} \Pi_{i}(H) F_{i 1}(E, H) \mathrm{d} H=1 \quad(i=1,2)
$$

and the functions $\Pi_{i}(1)(i=1,2)$ can be given as

$$
\Pi_{i}(1)=1+\frac{\pi^{2}}{48}\left(\frac{c}{k_{i} h}\right)^{2}-\frac{7 \pi^{4}}{5120}\left(\frac{c}{k_{i} h}\right)^{2}+\mathrm{O}\left(\frac{c}{h}\right)^{6} \quad\left(\frac{c}{h} \ll 1\right)
$$


We may obtain the solution of a strip containing a central impermeable crack perpendicular to its edges by letting $h \rightarrow \infty$. In this case, the corresponding Fredholm integral equation becomes

$$
\Pi_{i}(E)+\int_{0}^{1} \Pi_{i}(H) F_{i 3}(E, H) \mathrm{d} H=1 \quad(i=1,2)
$$

where

$$
F_{i 3}(E, H)=H \int_{0}^{\infty} \frac{1}{k_{i}^{2}} S\left[1-c t h\left(S b / k_{i} c\right)\right] I_{0}\left(S H / k_{i}\right) I_{0}\left(S E / k_{i}\right) \mathrm{d} S
$$

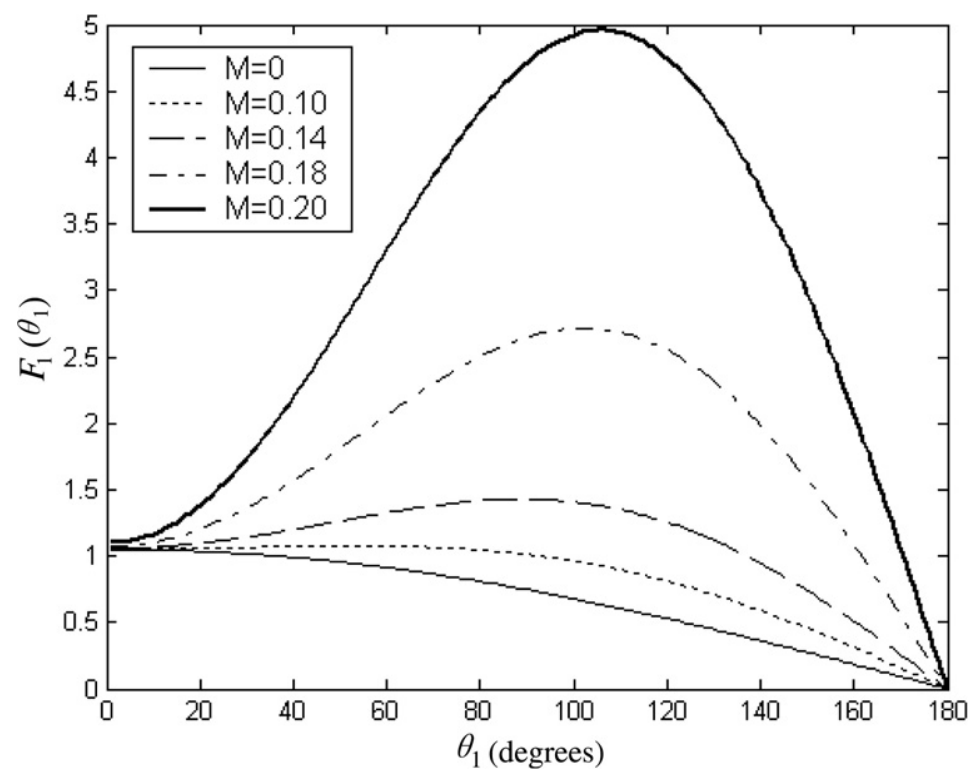

Fig. 2. $F_{1}\left(\theta_{1}\right)$ versus $\theta_{1}$ when $0 \leqslant M<M_{d}$ for permeable crack case.

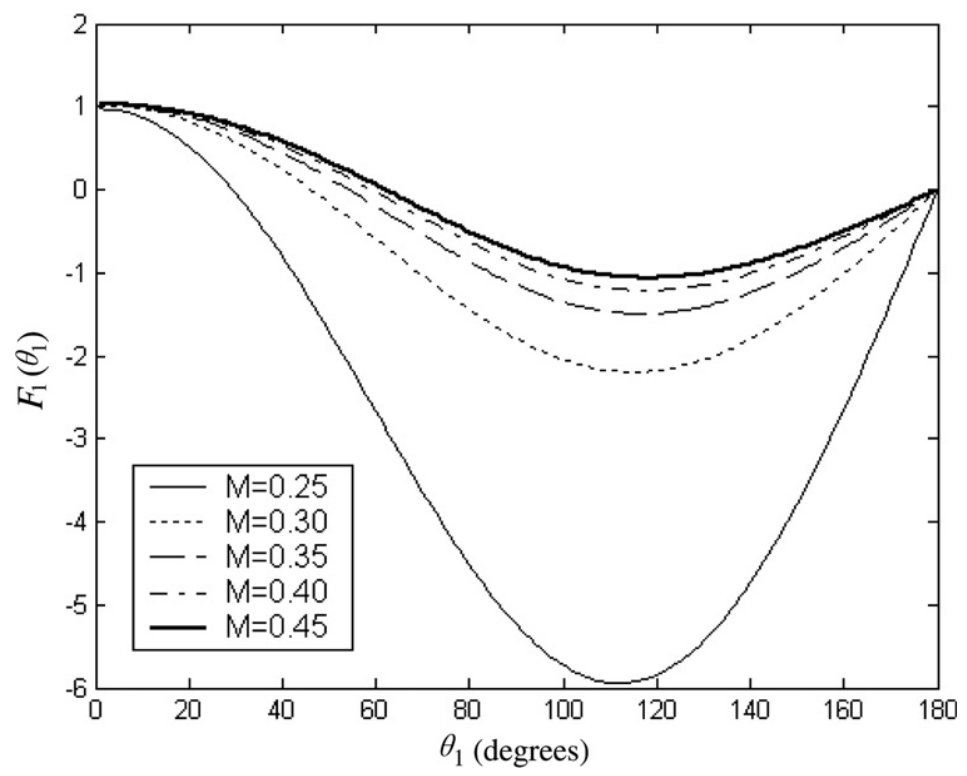

Fig. 3. $F_{1}\left(\theta_{1}\right)$ versus $\theta_{1}$ when $M_{d}<M<M_{\mathrm{c} 2}$ for permeable crack case. 
and $k_{i}(i=1,2)$ was defined in Eq. (60). The functions $\Pi_{i}(1)(i=1,2)$ in this case are defined as

$$
\Pi_{i}(1)=1+\frac{\pi^{2}}{24}\left(\frac{c}{b}\right)^{2}-\frac{19 \pi^{4}}{5760}\left(\frac{c}{b}\right)^{4}+\mathrm{O}\left(\frac{c}{b}\right)^{6} \quad\left(\frac{c}{b} \ll 1\right)
$$

The Fredholm integral equations (48) and (57) can also be solved by computer with the use of Gaussian quadrature formulas. Once this is done, the field intensity factors can be found from Eqs. (67)-(71) and (76)-(81).

Consider a transversely isotropic material exhibiting full coupling between elastic, electric, and magnetic fields, with a unique axis along the $x_{3}$-direction. The material constants we used are given as [5]:

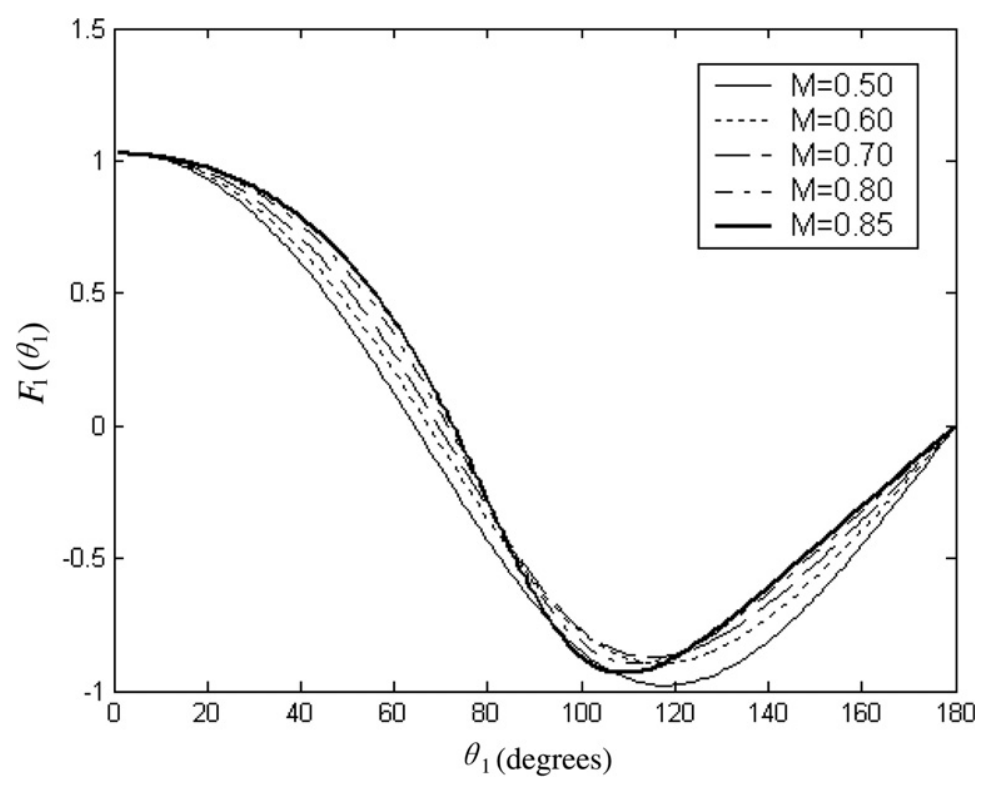

Fig. 4. $F_{1}\left(\theta_{1}\right)$ versus $\theta_{1}$ when $M_{\mathrm{c} 2}<M<M_{\mathrm{c} 3}$ for permeable crack case.

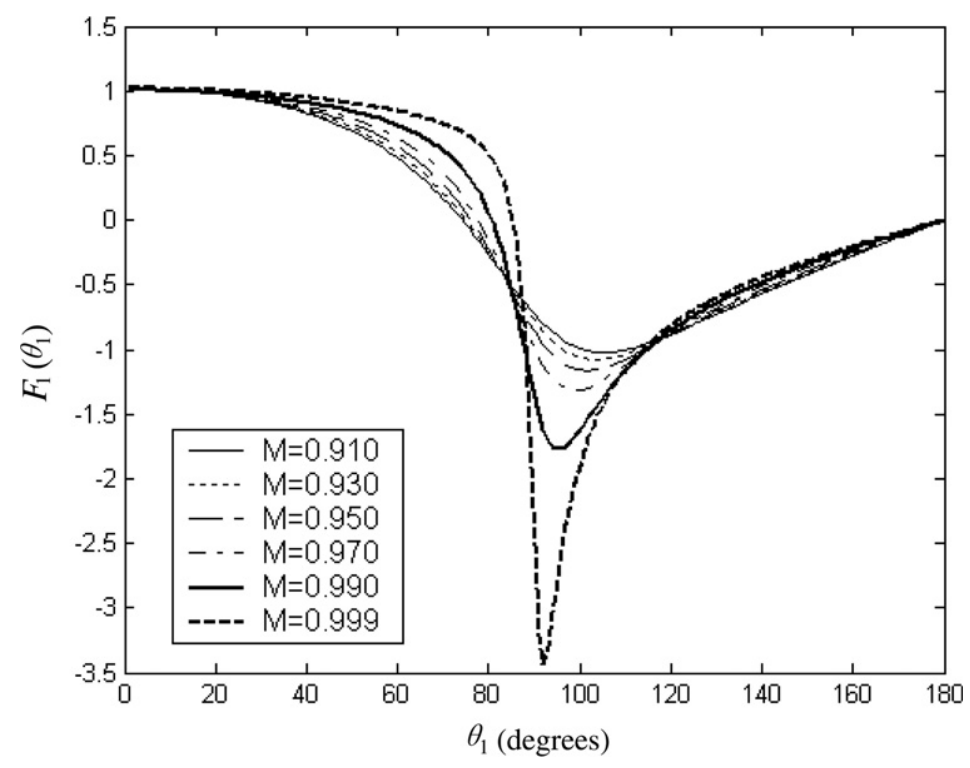

Fig. 5. $F_{1}\left(\theta_{1}\right)$ versus $\theta_{1}$ when $M_{\mathrm{c} 3}<M<1$ for permeable crack case. 


$$
\begin{aligned}
& c_{44}=4.53 \times 10^{10}\left(\mathrm{~N} / \mathrm{m}^{2}\right), \quad e_{15}=11.6\left(\mathrm{C} / \mathrm{m}^{2}\right), \quad h_{15}=550 \mathrm{~N} / \mathrm{A} \mathrm{m} \\
& \lambda_{11}=0.8 \times 10^{-10}\left(\mathrm{C}^{2} / \mathrm{N} \mathrm{m}^{2}\right), \quad \epsilon_{11}=5.9 \times 10^{-4}\left(\mathrm{~N} \mathrm{~s}^{2} / \mathrm{C}^{2}\right) \\
& \beta_{11}=0.5 \times 10^{-11}(\mathrm{~N} \mathrm{~s} / \mathrm{VC})
\end{aligned}
$$

By analyzing the extreme value of the function $F_{1}\left(\theta_{1}\right)$, we find the Mach number exhibits a critical value when $M_{\mathrm{c} 1}=0.087$. While $0 \leqslant M \leqslant M_{\mathrm{cl}}$ and $0^{\circ} \leqslant \theta_{1} \leqslant 180^{\circ}, F_{1}\left(\theta_{1}\right)$ decreases monotonically with increase of $\theta_{1}$ (see Fig. 2). The maximum value of the DSIF $K^{\mathrm{T}}\left(v, \theta_{1}\right)$ occurs at the crack axis $\theta_{1}=0^{\circ}$. This means that the crack has a tendency to propagate along its original plane when the criterion of the maximum tensile stress is used. For the case of $M_{\mathrm{c} 1}<M<M_{d}$ (while $\left.q \rightarrow \infty, M \rightarrow M_{d}=0.2275\right)$ and $0^{\circ} \leqslant \theta_{1} \leqslant 180^{\circ}, F_{1}\left(\theta_{1}\right)$ increases with the increase of $\theta_{1}$ at first and then decreases after it reaches a certain peak value. It is shown that the orientation of the maximum DSIF makes a branch angle of $\theta_{b}$ with the crack axis, and the higher the crack propagation speed, the larger the branch angle. This conclusion is in agreement with that obtained by $\mathrm{Hu}$ and $\mathrm{Li}$ [17] when our solution reduces to the infinite magnetoelectroelastic material case.

\begin{tabular}{|c|c|c|c|c|c|c|}
\hline \multirow[t]{2}{*}{$M$} & \multicolumn{6}{|l|}{$\theta_{1}$} \\
\hline & $0^{\circ}$ & $30^{\circ}$ & $60^{\circ}$ & $90^{\circ}$ & $120^{\circ}$ & $F_{1}\left(\theta_{b}\right)$ \\
\hline $0\left(\theta_{b}=0^{\circ}\right)$ & 1.0503 & 1.0141 & 0.9080 & 0.7394 & 0.5198 & 1.0503 \\
\hline $0.1\left(\theta_{b}=53^{\circ}\right)$ & 1.0538 & 1.0661 & 1.0740 & 1.0076 & 0.8011 & 1.0759 \\
\hline $0.2\left(\theta_{b}=105^{\circ}\right)$ & 1.1028 & 1.7750 & 3.3639 & 4.7437 & 4.7199 & 4.9585 \\
\hline $0.22\left(\theta_{b}=108^{\circ}\right)$ & 1.3183 & 4.8589 & 13.3538 & 21.0835 & 21.8565 & 22.6464 \\
\hline $0.25\left(\theta_{b}=111^{\circ}\right)$ & 0.9713 & -0.1159 & -2.7705 & -5.3003 & -5.8139 & -5.9442 \\
\hline $0.45\left(\theta_{b}=117^{\circ}\right)$ & 1.0302 & 0.7595 & 0.0316 & -0.7721 & -1.0614 & -1.0651 \\
\hline $0.5\left(\theta_{b}=0^{\circ}\right)$ & 1.0312 & 0.7824 & 0.0940 & -0.6924 & -0.9760 & 1.0312 \\
\hline $0.85\left(\theta_{b}=0^{\circ}\right)$ & 1.0260 & 0.8913 & 0.3681 & -0.6708 & -0.8628 & 1.0260 \\
\hline $0.9\left(\theta_{b}=0^{\circ}\right)$ & 1.0213 & 0.9083 & 0.4366 & -0.7443 & -0.8699 & 1.0213 \\
\hline $0.95\left(\theta_{b}=101^{\circ}\right)$ & 1.0124 & 0.9264 & 0.5443 & -0.9088 & -0.8685 & -1.1637 \\
\hline
\end{tabular}

Table 1

Values of $F_{1}\left(\theta_{1}\right)$ against $M$ and maximum value $F_{1}\left(\theta_{b}\right)$ for permeable crack case

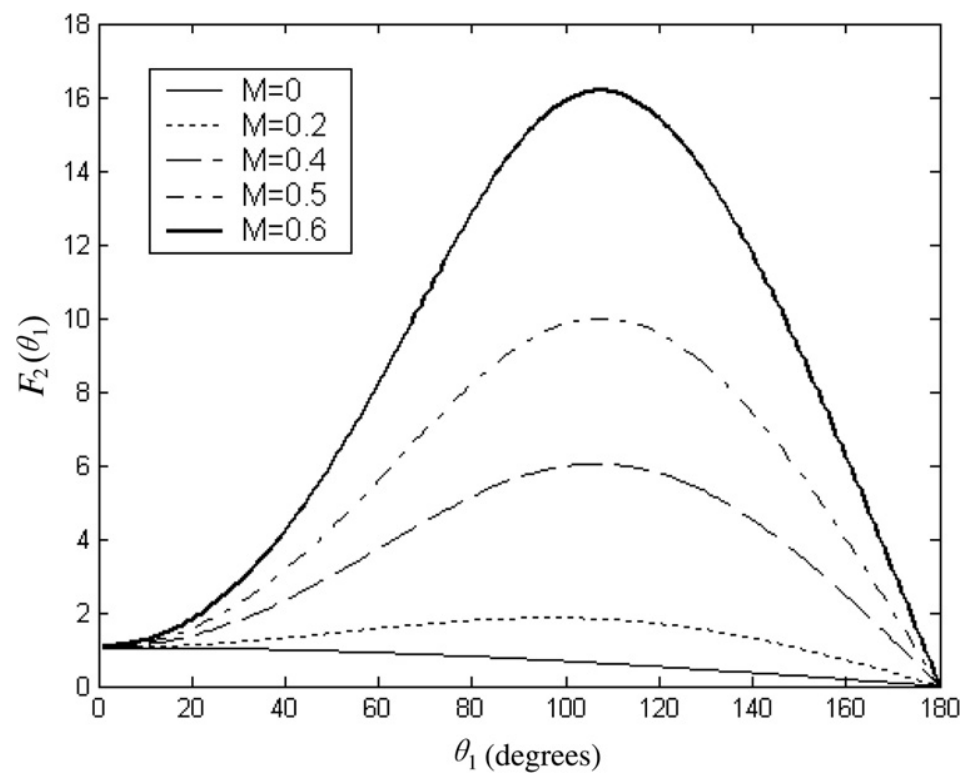

Fig. 6. $F_{2}\left(\theta_{1}\right)$ versus $\theta_{1}$ for impermeable crack case. 
For the case of $M>M_{d}$ and $0^{\circ} \leqslant \theta_{1} \leqslant 180^{\circ}$, the Mach number also exhibits two critical values when $M_{\mathrm{c} 2}=0.468$ and $M_{\mathrm{c} 3}=0.908$. From Fig. 3, it can be seen that the maximum magnitude of $F_{1}\left(\theta_{1}\right)$ occurs at an angle $\theta_{1} \neq 0^{\circ}$ when $M_{d}<M<M_{\mathrm{c} 2}$. This means that the crack will deviate from its original plane. At higher crack velocity, the maximum magnitudes of $F_{1}\left(\theta_{1}\right)$ always occur at angle $\theta_{1}=0^{\circ}$ when $M_{\mathrm{c} 2}<M<M_{\mathrm{c} 3}$. This means that the crack will propagate along its original plane, see Fig. 4. At higher Mach numbers $M_{\mathrm{c} 3}<M<1$, the crack will deviate from the original plane $\theta_{1}=0^{\circ}$, as shown in Fig. 5 .

Some results are listed in Table 1 for the magnitudes of $F_{1}\left(\theta_{1}\right)$ and the branch angle $\theta_{b}$ when $M$ varies near the three critical values $M_{c i}(i=1,2,3)$. It should be noted that in the above discussion we have used the finite geometry size of the rectangular body as $h / c=4, b / c=3$, without loss of generality.

By calculating the extreme values of $F_{2}\left(\theta_{1}\right)$ for the impermeable crack case, we obtain the critical Mach number, $M_{\mathrm{c}}=0.083$. At lower mach numbers $M \leqslant M_{\mathrm{c}}, F_{2}\left(\theta_{1}\right)$ monotonically decrease with the increase of $\theta_{1}$ (see Fig. 6); In the case of $M_{\mathrm{c}}<M<1$, the function $F_{2}\left(\theta_{1}\right)$ first increases with the increase of $\theta_{1}$ and then

Table 2

Values of $F_{2}\left(\theta_{1}\right)$ against $M$ and maximum value $F_{2}\left(\theta_{b}\right)$ for impermeable crack case

\begin{tabular}{|c|c|c|c|c|c|c|}
\hline \multirow[t]{2}{*}{$M$} & \multicolumn{6}{|l|}{$\theta_{1}$} \\
\hline & $0^{\circ}$ & $30^{\circ}$ & $60^{\circ}$ & $90^{\circ}$ & $120^{\circ}$ & $F_{2}\left(\theta_{b}\right)$ \\
\hline $0.6\left(\theta_{b}=106^{\circ}\right)$ & 1.0875 & 2.9470 & 8.4760 & 14.9217 & 15.3023 & 16.1716 \\
\hline $0.65\left(\theta_{b}=106^{\circ}\right)$ & 1.1058 & 3.3236 & 10.2640 & 18.9339 & 19.4050 & 20.6181 \\
\hline $0.7\left(\theta_{b}=106^{\circ}\right)$ & 1.1358 & 3.7518 & 12.4354 & 24.2339 & 24.7558 & 26.5085 \\
\hline $0.75\left(\theta_{b}=105^{\circ}\right)$ & 1.1881 & 4.2457 & 15.1154 & 31.5217 & 31.9775 & 34.6350 \\
\hline $0.8\left(\theta_{b}=104^{\circ}\right)$ & 1.2868 & 4.8349 & 18.5047 & 42.1584 & 42.2272 & 46.5249 \\
\hline $0.85\left(\theta_{b}=103^{\circ}\right)$ & 1.4947 & 5.5909 & 22.9608 & 59.2195 & 57.9679 & 65.6380 \\
\hline $0.9\left(\theta_{b}=101^{\circ}\right)$ & 2.0144 & 6.7374 & 29.2532 & 91.6022 & 85.7607 & 101.9269 \\
\hline $0.95\left(\theta_{b}=99^{\circ}\right)$ & 3.8143 & 9.3318 & 39.8752 & 181.8718 & 153.2221 & 202.8757 \\
\hline $0.97\left(\theta_{b}=97^{\circ}\right)$ & 6.0844 & 12.0737 & 47.8126 & 295.4318 & 225.9347 & 329.2586 \\
\hline $0.99\left(\theta_{b}=94^{\circ}\right)$ & 14.0501 & 21.0273 & 66.8716 & 830.7227 & 497.7983 & 917.8544 \\
\hline
\end{tabular}

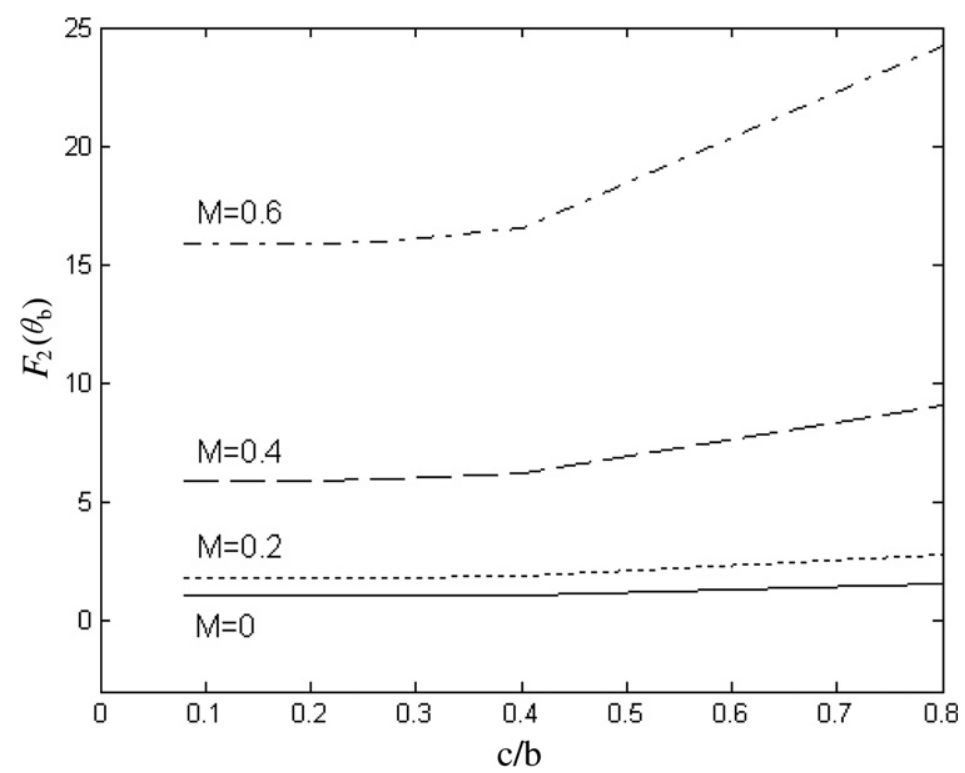

Fig. 7. $F_{2}\left(\theta_{b}\right)$ versus $c / b$ for different Mach numbers in impermeable crack case. 


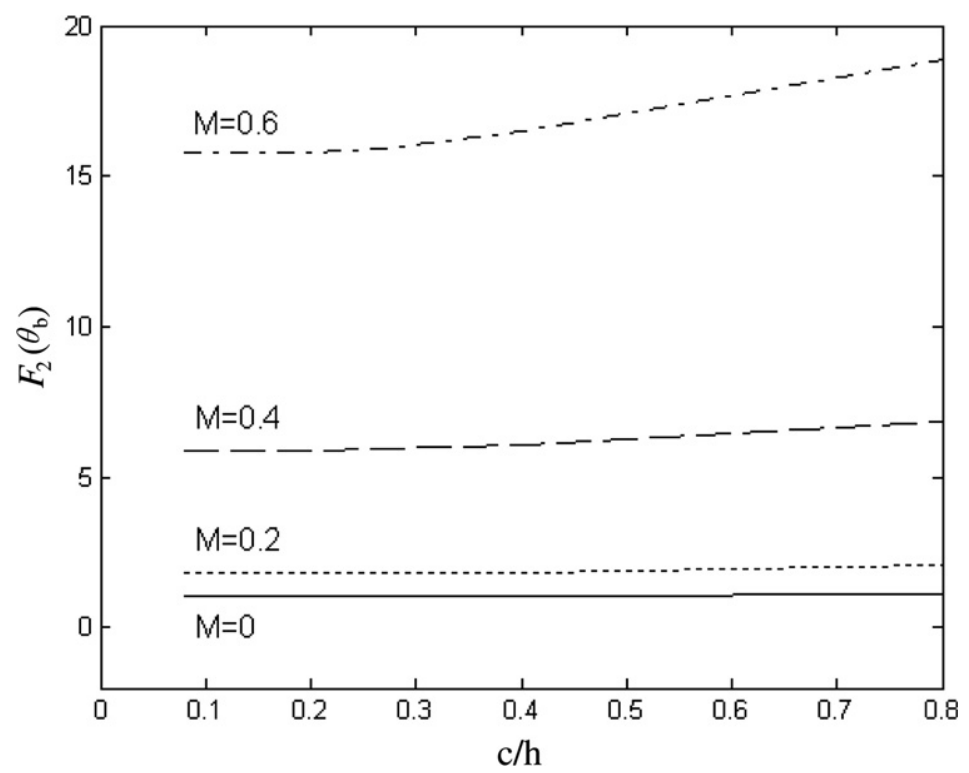

Fig. 8. $F_{2}\left(\theta_{b}\right)$ versus $c / h$ for different Mach numbers in impermeable crack case.

decreases after it reaches a certain peak value. Some results for the magnitudes of $F_{2}\left(\theta_{1}\right)$ and the branch angle $\theta_{b}$ for higher Mach numbers are listed in Table 2.

To study boundary effect of the rectangular domain with side lengths $2 b$ and $2 h$, different ratios of $c / b$ and $c / h$ of the rectangular domain are considered and Figs. 7 and 8 exhibit the variation of the function $F_{2}\left(\theta_{b}\right)$ against the length ratio $c / b$ and $c / h$ with fixed $h(h / c=4)$ and $b(b / c=4)$ values, respectively. It is evident that the function $F_{2}\left(\theta_{b}\right)$ increases with the increase of the $c / b$ and $c / h$ at fixed $h$ and $b$ values, respectively. The effects of the length ratio $c / b$ on the function $F_{2}\left(\theta_{b}\right)$ are greater than those of the length ratio $c / h$. It is also found from the two figures that the results from the proposed solution are almost the same as those from the solution of the same cracked body but with infinite domain when both $c / b$ and $c / h$ are less than 0.4 .

\section{Concluding remarks}

The magnetoelectroelastic problem of a finite crack in a rectangular magnetoelectroelastic body under longitudinal shear has been analyzed theoretically. A closed-form solution to the anti-plane crack problem has been obtained. Explicit expressions of field variables and the field intensity factors for permeable or impermeable cracks under eight possible loading conditions are derived. The stresses, electric displacements and magnetic inductions at the crack tip exhibit inverse square root singularities. In the case of permeable crack conditions, the crack will propagate along its original plane when $0 \leqslant M \leqslant M_{\mathrm{c} 1}$ and $M_{\mathrm{c} 2}<M<M_{\mathrm{c} 3}$, whereas in the range of $M_{\mathrm{c} 1}<M<M_{d}, M_{d}<M<M_{\mathrm{c} 2}$ and $M_{\mathrm{c} 3}<M<1$, the propagation of the crack possibly brings about the branching phenomenon. For the impermeable crack case, the crack will deviate from the original plane when $M_{\mathrm{c}}<M<1$. Numerical results show that the velocity of the crack and the geometry size of the rectangular body significantly influence the crack branching behavior.

\section{Acknowledgements}

The authors are grateful for the financial support of Foundation for Young Teachers in Tianjin University, and the reviewers for their valuable comments in improving the paper. 


\section{Appendix}

The constants $a_{0}, b_{0}$ and $c_{0}$ can be obtained by considering the edge loading conditions as

Case 1: $\left(\begin{array}{l}a_{0} \\ b_{0} \\ c_{0}\end{array}\right)=\left(\begin{array}{ccc}\mu & -e_{15} & -h_{15} \\ 0 & \lambda_{11} & \beta_{11} \\ 0 & \beta_{11} & \epsilon_{11}\end{array}\right)^{-1}\left(\begin{array}{c}P_{0} \\ D_{0} \\ B_{0}\end{array}\right)$

Case 2: $a_{0}=\gamma_{0}, \quad b_{0}=E_{0}-m_{1} \gamma_{0}, \quad c_{0}=\frac{1}{\epsilon_{11}}\left[B_{0}+\beta_{11}\left(m_{1} \gamma_{0}-E_{0}\right)\right]$

Case 3: $\left(\begin{array}{c}a_{0} \\ b_{0} \\ c_{0}\end{array}\right)=\left(\begin{array}{ccc}m_{1} & 1 & 0 \\ 0 & \beta_{11} & \epsilon_{11} \\ \mu & -e_{15} & -h_{15}\end{array}\right)^{-1}\left(\begin{array}{c}E_{0} \\ B_{0} \\ P_{0}\end{array}\right)$

$a_{0}=\gamma_{0}$

Case 4: $\left(\begin{array}{l}b_{0} \\ c_{0}\end{array}\right)=\left(\begin{array}{ll}\lambda_{11} & \beta_{11} \\ \beta_{11} & \epsilon_{11}\end{array}\right)^{-1}\left(\begin{array}{c}D_{0} \\ B_{0}\end{array}\right)$

Case 5: $\left(\begin{array}{l}a_{0} \\ b_{0} \\ c_{0}\end{array}\right)=\left(\begin{array}{ccc}\mu & -e_{15} & -h_{15} \\ 0 & \lambda_{11} & \beta_{11} \\ m_{2} & 0 & 1\end{array}\right)^{-1}\left(\begin{array}{l}P_{0} \\ D_{0} \\ H_{0}\end{array}\right)$

Case 6: $a_{0}=\gamma_{0}, \quad b_{0}=E_{0}-m_{1} \gamma_{0}, \quad c_{0}=H_{0}-m_{2} \gamma_{0}$

Case 7: $\left(\begin{array}{c}a_{0} \\ b_{0} \\ c_{0}\end{array}\right)=\left(\begin{array}{ccc}\mu & -e_{15} & -h_{15} \\ m_{1} & 1 & 0 \\ m_{2} & 0 & 1\end{array}\right)^{-1}\left(\begin{array}{c}P_{0} \\ E_{0} \\ H_{0}\end{array}\right)$

Case 8: $a_{0}=\gamma_{0}, \quad b_{0}=\frac{1}{\lambda_{11}}\left[D_{0}+\beta_{11}\left(m_{2} \gamma_{0}-H_{0}\right)\right], \quad c_{0}=H_{0}-m_{2} \gamma_{0}$

\section{References}

[1] Wu TL, Huang JH. Closed-form solutions for the magnetoelectric coupling coefficient in fibrous composites with piezoelectric and piezomagnetic phases. Int J Solids Struct 2000;37:2981-3009.

[2] Zhou ZG, Wu LZ, Wang B. The dynamic behavior of two collinear interface cracks in magneto-electro-elastic composites. Eur J Mech A/Solids 2005;24:253-62.

[3] Nan CW. Magnetoelectric effect in composites of piezoelectric and piezomagnetic phases. Phys Rev B 1994;50:6082-8.

[4] Huang JH, Liu HK, Dai WL. The optimized fiber volume fraction for magnetoelectric coupling effect in piezoelectric-piezomagnetic continuous fiber reinforced composites. Int J Engng Sci 2000;38:1207-17.

[5] Li JY. Magnetoelectroelastic multi-inclusion and inhomogeneity problems and their applications in composite materials. Int J Engng Sci 2000;38:1993-2011.

[6] Soh AK, Liu JX, Hoon KH. Three-dimensional Green's functions for transversely isotropic magnetoelectroelastic solids. Int J NonLinear Sci Numer Simul 2003;4:139-48.

[7] Wang X, Shen YP. Inclusions of arbitrary shape in magnetoelectroelastic composite materials. Int J Engng Sci 2003;41:85-102.

[8] Wang $\mathrm{X}$, Zhong Z. A circular tube or bar of cylindrically anisotropic magnetoelectroelastic material under pressuring loading. Int $\mathbf{J}$ Engng Sci 2003;41:2143-59.

[9] Song ZF, Sih GC. Crack initiation behavior in a magnetoelectroelastic composite under in-plane deformation. Theor Appl Fract Mech 2003;39:189-207.

[10] Gao CF, Hannes K, Herbert B. Crack problems in magnetoelectroelastic solids: Part I. Exact solution of a crack. Int J Engng Sci 2003;41:969-81.

[11] Wang BL, Mai YW. Crack tip field in piezoelectric/piezomagnetic media. Eur J Mech A/Solids 2003;22:591-602.

[12] Wang BL, Mai YW. Fracture of piezoelectromagnetic materials. Mech Res Comm 2004;31:65-73.

[13] Zhou ZG, Wang B, Sun YG. Two collinear interface cracks in magneto-electro- elastic composites. Int J Engng Sci 2004;42:1155-67. 
[14] Qin QH. 2D Green's functions of defective magnetoelectroelastic solids under thermal loading. Engng Anal Boundary Elements 2005;29(6):577-85.

[15] Li XF. Dynamic analysis of a cracked magnetoelectroelastic medium under antiplane mechanical and inplane electric and magnetic impacts. Int J Solids Struct 2005;42(11-12):3185-205.

[16] Sih GC, Yu HY. Volume fraction effect of magnetoelectroelastic composite on enhancement and impediment of crack growth. Composite Struct 2005;68(1):1-11.

[17] $\mathrm{Hu} \mathrm{KQ}$, Li GQ. Constant moving crack in a magnetoelectroelastic material under anti-plane shear loading. Int $\mathbf{J}$ Solids Struct 2005;42(9-10):2823-35.

[18] Yoffe EH. The moving Griffith crack. Phil Mag 1951;42:739-50.

[19] Sih GC, Chen EP. Moving cracks in layered composites. Int J Engng Sci 1982;20(11):1181-92.

[20] Dhaliwal RS, He WH, Saxena HS, Rokne JG. A moving Griffith crack at the interface of two dissimilar elastic layers. Engng Fract Mech 1992;43(6):923-30.

[21] Das S, Patra B. Moving Griffith crack at the interface of two dissimilar orthotropic half planes. Engng Fract Mech 1996;54(4):523-31.

[22] Chen ZT, Karihaloo BL, Yu SW. A Griffith crack moving along the interface of two dissimilar piezoelectric materials. Int J Fract 1998;91:197-203.

[23] Kwon SM, Lee KY. Constant moving crack in a piezoelectric block: anti-plane problem. Mech Mater 2001;33:649-57.

[24] Kwon SM, Lee KY. Steady state crack propagation in a piezoelectric layer bonded between two orthotropic layers. Mech Mater 2003;35(11):1077-88.

[25] Kwon SM. On the dynamic propagation of an anti-plane shear crack in a functionally graded piezoelectric strip. Acta Mech 2004;167(1-2):73-89.

[26] Li C, Weng GJ. Yoffe-type moving crack in a functionally graded piezoelectric material. Proc Royal Soc Lond A 2002;458:381-99.

[27] Parton VZ, Kudryurtsev BA. Electromagnetoelasticity. New York: Gordon and Breach; 1998.

[28] Zhang XS. Solution of a rectangular sheet containing a central crack propagating at constant velocity under antiplane shear. Engng Fract Mech 1985;22:181-8.

[29] Kwon SM, Lee KY. Analysis of stress and electric fields in a rectangular piezoelectric body with a center crack under anti-plane shear loading. Int J Solids Struct 2000;37:4859-69.

[30] Sneddon IN. Application of integral transforms in the theory of elasticity. Vienna: Springer; 1975.

[31] Magnus W, Oberhettinger F, Soni RP. Formulas and theorems for the special functions of mathematical physics. New York: Springer; 1966.

[32] $\mathrm{Hu} \mathrm{KQ,} \mathrm{Li} \mathrm{GQ.} \mathrm{Electro-magneto-elastic} \mathrm{analysis} \mathrm{of} \mathrm{a} \mathrm{piezoelectromagnetic} \mathrm{strip} \mathrm{with} \mathrm{a} \mathrm{finite} \mathrm{crack} \mathrm{under} \mathrm{longitudinal} \mathrm{shear.} \mathrm{Mech}$ Mater 2005;37:925-34. 